\title{
Stamps extraction using local adaptive k-means and ISODATA algorithms
}

\author{
Maha A. Rajab ${ }^{1}$, Loay E. George ${ }^{2}$ \\ ${ }^{1}$ College of Information Technology, University of Babylon, Baghdad, Iraq \\ ${ }^{2}$ University of Information Technology and Communication, Baghdad, Iraq
}

\begin{abstract}
Article Info
Article history:

Received Apr 19, 2020

Revised Jul 15, 2020

Accepted Jul 27, 2020

Keywords:

Image segmentation

ISODATA

K-Mean

Region growing

ABSTRACT

One of the main difficulties facing the certified documents documentary archiving system is checking the stamps system, but, that stamps may be contains complex background and surrounded by unwanted data. Therefore, the main objective of this paper is to isolate background and to remove noise that may be surrounded stamp. Our proposed method comprises of four phases, firstly, we apply k-means algorithm for clustering stamp image into a number of clusters and merged them using ISODATA algorithm. Secondly, we compute mean and standard deviation for each remaining cluster to isolate background cluster from stamp cluster. Thirdly, a region growing algorithm is applied to segment the image and then choosing the connected region to produce a binary mask for the stamp area. Finally, the binary mask is combined with the original image to extract the stamp regions. The results indicate that the number of clusters can be determined dynamically and the largest cluster that has minimum standard deviation (i.e., always the largest cluster is the background cluster). Also, show that the binary mask can be established from more than one segment to cover are all stamp's disconnected pieces and it can be useful to remove the noise appear with stamp region.
\end{abstract}

This is an open access article under the $\underline{C C B Y-S A}$ license.

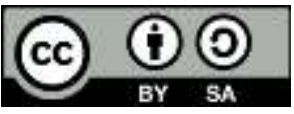

Corresponding Author:

Maha A. Rajab

College of Information Technology

University of Babylon, Baghdad, Iraq

Email: maabd444@gmail.com

\section{INTRODUCTION}

Although the massive use of computer technology in numerous fields of our lives. The performance of paper documents still plays a significant role. Contracts, wills, certificates, invoices and all documents issued by the official authorities are printed on hard paper and a signature or stamp that assure the authenticity of the content [1-4]. Thus, stamping is the process of locating the stamps on paper documents which hold a certain property such as (shape, complexity, background and typical patterns) [5, 6]. The main objective of utilizing a stamp is to certify a document for various kinds of verification, such as authentication, authorization, etc. [7]. There is a need to organize and access digitized documents according to their contents in processing the image of the document. Most official documents scanned be composed of graphics such as stamps. The legitimacy of the document is provided by a stamp [8,9]. Document scanning is a method for changing printed documents in digital form. There is a common problem encountered when scanning documents is the "noise" that can occur in the image because of the type of the paper, printing machine used, or they can be created through the scanners during the scanning process. One step in the pre-processing is noise removal. Between other things, noise, minimizes the quality of next tasks $[10,11]$. Image segmentation 
is the process of dividing an image into parts or objects composing it in the image, i.e. the group of pixels or pixels in a similar area depending on some homogeneity criteria such as color, density, or texture, to define the border in an image [12-14]. Image clustering is one of the best methods that can be used for segmentation of images. The clustering algorithm aims to improve the partitioning decisions depending on a set of primary clusters, which are updated after each iteration $[15,16]$.

There are several forms of clustering: hierarchical clustering, Fuzzy C-means clustering, K-means clustering and Iterative Self-Organizing Data Analysis Technique Algorithm (ISODATA). The K-means method is one of the most generally used clustering techniques for various applications [17-20]. ISODATA is identical to k-means in which that reduced the number of clusters during the process of reassignment and update $[21,22]$. In ISODATA, if the clusters centers have a distance low than a certain threshold, the clusters are merged. Inversely, split the cluster into two clusters. Finally, it is obtained a result of the final clustering process when accessed to all the specified conditions [23, 24].

The problems facing the process of extraction stamps from documents, is that, in general, during the process of capturing an image of the document and cropping stamp from it, and due to the lighting changes resulting from the capturing, the stamp can be placed on complex background, the background color is not white and may contain gradations of colors, some background colors which may be close to the stamp color, so it is necessary to isolate the background from the foreground. Also, another problem, the stamps may be surrounded by unwanted data such as word, text, patches and other types of noise which is formed as object nearest the stamps.

This paper aims to present a new approach for isolation background from foreground and also removing objects around the stamp such as (noise, word, text and etc.) and stamps which are in different shapes as well as different sizes. The suggested system could used as a tool for extracting stamps of different colors, shapes, size and direction.

This paper is organized as follows, Section 2 introduces the layout of proposed method. Section 3 describes the used stamps dataset for performance evaluation. Section 4 illustrates the results and discussion of conducted tests. The derived conclusion are shown in Section 5.

\section{PROPOSED METHOD}

In this section, an efficient and robust method for the extraction color stamp is presented. The goal of the proposed method is to isolate the background from the foreground or stamp and delete the noise and unwanted data such as word, text and patches which is formed as object nearest the stamps. Figure 1 illustrate the layout of the proposed method of this paper that consists of many stages.

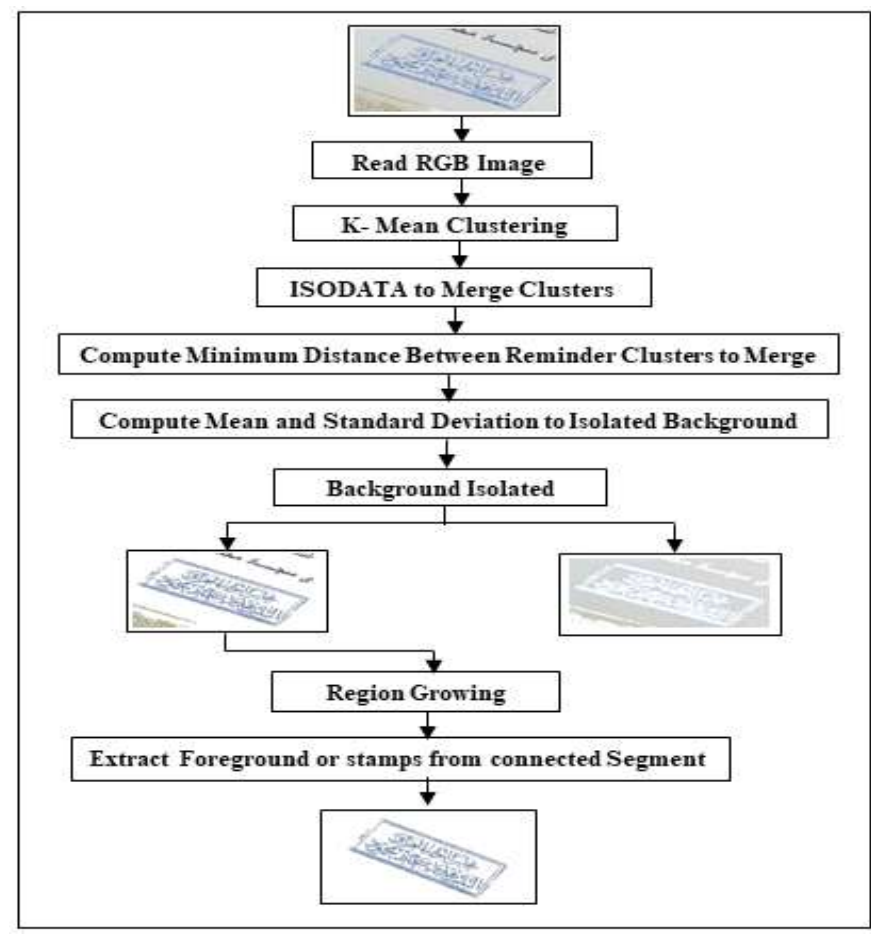

Figure 1. The layout of the proposed stamp extraction 


\subsection{Load stamp image}

In image processing, the utilization of color is useful because the color has influential descriptor that simplifies object extraction. Colors are seen as mixtures of the primary colors of light: Red (R), Green (G) and Blue (B). The proposed method starts from reading the input RGB image from stamps dataset which is described below. Stamp image is the bitmap (i.e., .bmp) image file. The color resolution of the stamp image is $24 \mathrm{bit} /$ pixel. The image data (i.e. R, G and B components) are loaded. After that, the loaded components which are used as the input image for k-mean clustering technique.

\subsection{K-Means clustering algorithm}

Segmentation is the process of dividing the image into a number of areas. Clustering is the process of grouping similar data objects that have the same color into the same cluster and data objects hold various colors to another various clusters. A clustering algorithm such as K-Means clustering algorithm attempts to group a set of data objects into $\mathrm{k}$ clusters depended on the distance between the data object and the $\mathrm{k}$ centroids chosen. The k-means algorithm starts with the following steps as [25]:

Step 1 : Select initial random centroid for each cluster. The initial step for k-means in the proposed method is to select four initial random centroids for each band of image (i.e. RGB) arranged respectively as $20,90,170$ and 230 . In this case, the number of probabilities for the values of the random centroids will become equal to $\mathrm{L}=4^{3}=64$.

Step 2 : Compute the difference between each data point (i.e. pixel) for each band of an image with each centroid as (1), (2) and (3) respectively.

$$
\begin{aligned}
& D_{R}=\text { Red }- \text { centroidR } \\
& D_{G}=\text { Green }- \text { centroidG } \\
& D_{B}=\text { Blue }- \text { centroidB }
\end{aligned}
$$

Where $\boldsymbol{D}_{\boldsymbol{R}}, \boldsymbol{D}_{\boldsymbol{G}}, \boldsymbol{D}_{\boldsymbol{B}}$, represent difference between data points and centroid for all three bands.

Step 3: Apply Euclidean distance to compute the summation of the squares differences between each pixel and its assigned centroid for each band of the image as (4).

$$
d_{E U C}=\sum_{i=1}^{n} D_{R}^{2}+D_{G}^{2}+D_{B}^{2}
$$

where $\mathrm{d}_{\mathrm{EUC}}$ represent the Euclidean Distance.

Step 4 : Find the minimum distance between clusters.

Step 5 : Grouping data points together, which have similar color depending on the minimum distance to assign the clusters.

Step 6: When clustering is finished, we need to compute the mean of the clusters determine the nearest centroid. At the time, the mean color is computed in each cluster to be remapped onto the image.

\subsection{ISODATA clustering algorithm}

Although K-Mean generated a huge number of clusters for each image and also the number of clusters $\mathrm{K}$ remains the same during the iteration. On the other hand, one of the aims of this paper is to automatically partition the input image into clusters using spatio-statistical criteria, and to isolate the background cluster from the foreground cluster. Therefore, we have to reduce the number of clusters by combining the similar using ISODATA clustering algorithm. The ISODATA clustering algorithm is similar in its principle to the K-Means algorithm. However, in the ISODATA algorithm the number of clusters is determined automatically through the iteration by merging similar clusters and splitting clusters based on thresolding value. The combination between K-mean algorithm and ISODATA algorithm to merged clusters involve the following steps as:

Step 1 : This step is the development step for k-mean which includes reducing the number of clusters for each image. This step starting after a limited number of iterations of k-mean, and this number is determined by user. Thus when the number of rounds becomes greater than three, the operation of the ISODATA algorithm begins.

Step 2 : ISODATA algorithm starts by relying on the number of clusters in the previous round, and calculates the minimum distance between the first cluster and the rest of the cluster, as well as the second cluster and the rest of the cluster, and so on to the rest of the other cluster.

Step 3 : The criteria for combining clusters are based on a specific threshold value and compared with minimum distance. The threshold value used in this paper is equal to 100. If the minimum distance 
is larger than the threshold value, the center of the cluster that carries the lowest distance and center of cluster zero will be update as (5), (6) and (7) respectively. The value of the new center becomes the center of the cluster with lowest distance, while the center of the cluster zero keeps its value.

$$
\begin{aligned}
& \mathrm{N}_{i}=\operatorname{count}[\mathrm{I}] \\
& \mathrm{N}_{j}=\operatorname{count}[\mathrm{J}] \\
& \text { Center }_{I}=\mathrm{N}_{i} * \operatorname{Center}_{I}+\mathrm{N}_{j} * \operatorname{Center}_{J} / \mathrm{N}_{i}+\mathrm{N}_{j}
\end{aligned}
$$

Where $\mathrm{N}_{\mathrm{i}}$ and $\mathrm{N}_{\mathrm{j}}$ represent the count of the cluster with lowest distance and cluster zero, while Center ${ }_{i}$ represents the update value for the center of the cluster.

Step 4 : After that, the number of the Cluster that carries the lowest distance will be combined with Cluster zero. Thus, the number of clusters will be decreased by one.

Step 5 : Repeat the above steps 2, 3, 4, 5 and 6 in section 2.2 until all the data points are completely passed. The data points do not transfer from one cluster to another. Thus, the clusters are stable and the clustering process finishes.

\subsection{Compute minimum distance}

At this stage, we need to calculate the distance between all the clusters produced from the previous stage. After that, we determine the two clusters that have the lowest distance and are combined depending on the color value of the pixels of the clusters selected for the merging. This process will be continuing to merge all clusters which have lowest distance. Thus, the number of the clusters will be reduced.

\subsection{Compute mean and standard deviation}

This stage aims to isolate the background clusters from the stamp clusters (i.e., extract the background from the remaining clusters in the previous stage). In this stage we need to compute mean and standard deviation for the remaining clusters. After that, the cluster that has the most repetition, (i.e., the larger Cluster) will have less standard deviation than the rest of the other cluster. Thus, this measure is helpful for determining the background from the stamp clusters. It is necessary to observe the largest cluster is not constantly the background cluster and other clusters may also comprise a background. Thus, we need to decide which of the remaining clusters contains a background. This is done by counting the repetition of each color value for each cluster and keeping the value of the largest repetition. From experience, we find that the cluster, which holds the largest repetition whose value reaches more than 10 , is considered this cluster of background clusters, but if less than 10 is regarded among the stamp clusters. As a result, we determined the number of clusters for background and stamp, these clusters will be merging to get one image for both the background and the stamp. The merging process is done by adding the color values for each band (i.e., RGB) of all the clusters and dividing it by the number of background clusters, also the same thing will occur for the stamp clusters. Thus, we guarantee to obtain a background image and an image of the stamp.

\subsection{Extract stamp and remove object}

This stage aims to perform the segmentation process on the stamp image to extract stamp and remove unwanted information. This stage consists of the following steps as:

Step 1 : The segmentation process was used region growing based on eight neighbors for segmented the image in to a set of points, called seeds. Region growing start with a number of seeds which have been grouped into $\mathrm{n}$ sets. It searches the seed point's neighbors to see if they belong to the same region. The process is performed repeatedly so no new neighbors can be added to the region. Finally, the result of segmentation is a number of segments, and each segment can be considered as an image.

Step 2 : Calculate the main diagonal and the secondary diagonal for each of the resulting segments, so that consist of four points, two of them for the main diagonal and two for the secondary diagonal. A segment that contains four connected points with it contains data is considered a stamp. While a segment that does not contain four connected points is considered noise. As a result, there is a number of connected segments.

Step 3 : The main aim to build mask from the connected segments is to remove the unwanted data that surrounding stamp. The mask is generated from the connected segments, which is the same size as the image. It is significant to note that, the mask will be in the form of binary image by changing the color values of the segments to 1 and the rest of other values to 0 . After building the mask, we need to create a vector with size equal to the width of the mask, if all the values of the vector equal to 0 , 
all the values of the vector become 0 , but, where the values, not equal to 0 , we need to find the minimum and maximum index of the vector to set the value of 1 between indexes of vector. Thus, we get a binary mask.

Step 4 : In the end, we multiply the binary mask with the original image after isolating the background, then we become a stamp image free from Noise, in other words, multiplied the color value by 1 , we will get the color value, but the value of 0 converted to 255 as the background.

\section{STAMPS DATASET}

The size of the dataset is $48.9 \mathrm{MB}$. It contains a total of 1557 color stamps samples which are stored as BMP image format. The images of stamps were acquired from documents using a scanner and mobile camera. The cropping process was applied to capture only the stamp area of the whole document, using three applications which are paint, snip and sketch software. For each cropped stamp, eight stamp images are produced from rotating the original one at different angles clockwise. Rotation starts from 5 degrees and increases by 5 degrees until it reaches to 40 degrees. Note the orientation of the original stamp image is with the horizon line, which means that it is at an angle of zero. The stamps have different size, shape, complexity, position, directions and colors. For example, there are six categories of stamp shapes arranged as circle, oval, square, rectangular, triangular and some other irregular shapes. It's worth to mention that the collected dataset may be degraded in quality and resolution and the stamp can be located on complex background. The dataset was collected from various sources, involving official documents from Iraqi educational institutions, documents from syndicates such as teachers, engineers, doctors, pharmacists, Iraqi hospitals, provincial councils, and most Iraqi institutions. The dataset also contains some stamps from other Arab countries. Stamps dataset is available on the site: https://data.mendeley.com/datasets/ktr99fc826/3, DOI: 10.17632/ktr99fc826.3

\section{RESULTS AND DISCUSSION}

One dataset has been employed for evaluating the performance of the proposed method as described in section 3. Table 1, Table 2, Table 3 and Table 4 demonstrate the results of applying k-mean clustering algorithm and merged with ISODATA clustering algorithm on one image named as $136 \mathrm{~g} 30$ from the dataset. The results indicate that when increased the number of rounds the number of cluster decreased, due to the merged clusters using ISODAT clustering, thus, the number of cluster determine dynamically.

Also, Figure 2 shows the result of clustered the same image before and after clustering (i.e., k-mean and ISODAT clustering algorithms). Table 5 presents the result of computing the minimum distance between clusters and merge them. Respectively, the mean and standard deviation computed to isolate background clusters of stamp clusters with noise as shown in Figure 3.

Figure 4 illustrates that the cluster of the stamp image with noise is segmented to a number of segments using a region growing technique that based on eight neighbors. Then, take the connected segments that have four points where two points for main diagonal and other two points for secondary diagonal to build binary mask, note that, in Figure 4 the segment (2) is used to generated binary mask and other segments will be considered noise or unwanted data.

Table 1. The result of clustering image for round 1,2 and 3

\begin{tabular}{|c|c|c|c|c|c|c|c|c|c|c|c|c|}
\hline $\begin{array}{l}\text { Cluster } \\
\text { Number }\end{array}$ & $\begin{array}{l}\text { Center } \\
\text { Red }\end{array}$ & $\begin{array}{l}\text { Center } \\
\text { Green }\end{array}$ & $\begin{array}{l}\text { Center } \\
\text { Blue }\end{array}$ & Count & $\begin{array}{c}\text { Center } \\
\text { Red }\end{array}$ & $\begin{array}{l}\text { Center } \\
\text { Green }\end{array}$ & $\begin{array}{l}\text { Center } \\
\text { Blue }\end{array}$ & Count & $\begin{array}{c}\text { Center } \\
\text { Red }\end{array}$ & $\begin{array}{l}\text { Center } \\
\text { Green }\end{array}$ & $\begin{array}{l}\text { Center } \\
\text { Blue }\end{array}$ & Count \\
\hline \multicolumn{5}{|c|}{ Number Round=1 } & \multicolumn{4}{|c|}{ Number Round=2 } & \multicolumn{4}{|c|}{ Number Round $=3$} \\
\hline 0 & 103 & 105 & 111 & 234 & 97 & 100 & 106 & 176 & 96 & 98 & 104 & 160 \\
\hline 1 & 116 & 126 & 142 & 80 & 114 & 125 & 144 & 76 & 115 & 128 & 149 & 96 \\
\hline 2 & 125 & 132 & 129 & 3 & 126 & 129 & 132 & 128 & 125 & 128 & 132 & 169 \\
\hline 3 & 126 & 138 & 158 & 191 & 136 & 146 & 165 & 615 & 139 & 149 & 168 & 736 \\
\hline 4 & 164 & 160 & 125 & 31 & 175 & 171 & 138 & 160 & 177 & 174 & 143 & 249 \\
\hline 5 & 166 & 173 & 179 & 3935 & 162 & 171 & 183 & 2345 & 160 & 170 & 187 & 1852 \\
\hline 6 & 181 & 191 & 209 & 2183 & 179 & 190 & 206 & 2178 & 179 & 189 & 205 & 2134 \\
\hline 7 & 198 & 203 & 189 & 92 & 196 & 200 & 191 & 184 & 197 & 199 & 191 & 377 \\
\hline 8 & 195 & 206 & 219 & 1617 & 196 & 206 & 220 & 2393 & 196 & 207 & 221 & 2657 \\
\hline 9 & 203 & 198 & 184 & 148 & 196 & 194 & 172 & 661 & 192 & 190 & 169 & 719 \\
\hline 10 & 204 & 199 & 205 & 18 & 205 & 207 & 206 & 1309 & 207 & 211 & 209 & 3165 \\
\hline 11 & 206 & 206 & 193 & 603 & 207 & 208 & 196 & 635 & 207 & 207 & 195 & 571 \\
\hline 12 & 213 & 217 & 219 & 16425 & 214 & 218 & 220 & 14700 & 215 & 219 & 221 & 12675 \\
\hline
\end{tabular}


Table 2. The result of clustering image for round 4, 5 and 6

\begin{tabular}{|c|c|c|c|c|c|c|c|c|c|c|c|c|}
\hline $\begin{array}{l}\text { Cluster } \\
\text { Number }\end{array}$ & $\begin{array}{c}\text { Center } \\
\text { Red }\end{array}$ & $\begin{array}{l}\text { Center } \\
\text { Green }\end{array}$ & $\begin{array}{c}\text { Center } \\
\text { Blue }\end{array}$ & Count & $\begin{array}{c}\text { Center } \\
\text { Red }\end{array}$ & $\begin{array}{l}\text { Center } \\
\text { Green }\end{array}$ & $\begin{array}{l}\text { Center } \\
\text { Blue }\end{array}$ & Count & $\begin{array}{c}\text { Center } \\
\text { Red }\end{array}$ & $\begin{array}{l}\text { Center } \\
\text { Green }\end{array}$ & $\begin{array}{l}\text { Center } \\
\text { Blue }\end{array}$ & Count \\
\hline \multicolumn{5}{|c|}{ Number Round=4 } & \multicolumn{4}{|c|}{ Number Round=5 } & \multicolumn{4}{|c|}{ Number Round $=6$} \\
\hline 0 & 95 & 97 & 103 & 153 & 121 & 134 & 154 & 165 & 108 & 113 & 121 & 352 \\
\hline 1 & 118 & 131 & 152 & 130 & 111 & 114 & 119 & 342 & 140 & 151 & 168 & 1017 \\
\hline 2 & 125 & 128 & 132 & 185 & 142 & 152 & 170 & 814 & 177 & 173 & 142 & 225 \\
\hline 3 & 141 & 151 & 169 & 791 & 177 & 173 & 142 & 223 & 160 & 172 & 190 & 1572 \\
\hline 4 & 177 & 174 & 144 & 252 & 160 & 171 & 189 & 1597 & 180 & 190 & 205 & 2214 \\
\hline 5 & 160 & 171 & 188 & 1758 & 180 & 190 & 205 & 2216 & 172 & 174 & 172 & 237 \\
\hline 6 & 179 & 189 & 205 & 2139 & 172 & 174 & 171 & 2216 & 197 & 207 & 221 & 2703 \\
\hline 7 & 198 & 199 & 191 & 459 & 197 & 207 & 221 & 2789 & 192 & 191 & 169 & 677 \\
\hline 8 & 197 & 207 & 221 & 2763 & 192 & 191 & 169 & 682 & 209 & 212 & 210 & 4279 \\
\hline 9 & 191 & 189 & 168 & 663 & 209 & 213 & 211 & 4826 & 204 & 204 & 194 & 985 \\
\hline 10 & 209 & 212 & 211 & 4329 & 204 & 204 & 195 & 1130 & 215 & 219 & 222 & 11299 \\
\hline 11 & 206 & 206 & 196 & 697 & 215 & 219 & 222 & 10575 & & & & \\
\hline 12 & 215 & 219 & 222 & 11241 & & & & & & & & \\
\hline
\end{tabular}

Table 3. The result of clustering image for round 7, 8 and 9

\begin{tabular}{|c|c|c|c|c|c|c|c|c|c|c|c|c|}
\hline $\begin{array}{l}\text { Cluster } \\
\text { Number }\end{array}$ & $\begin{array}{l}\text { Center } \\
\text { Red } \\
\text { Number }\end{array}$ & $\begin{array}{l}\text { Center } \\
\text { Green } \\
\text { ound=7 }\end{array}$ & $\begin{array}{c}\text { Center } \\
\text { Blue }\end{array}$ & Count & $\begin{array}{c}\text { Center } \\
\text { Red } \\
\text { Numbe }\end{array}$ & $\begin{array}{l}\text { Center } \\
\text { Green } \\
\text { Round }=8\end{array}$ & $\begin{array}{l}\text { Center } \\
\text { Blue }\end{array}$ & Count & $\begin{array}{c}\text { Center } \\
\text { Red } \\
\text { Numbe }\end{array}$ & $\begin{array}{l}\text { Center } \\
\text { Green } \\
\text { Round=c }\end{array}$ & $\begin{array}{l}\text { Center } \\
\text { Blue }\end{array}$ & Count \\
\hline 0 & 131 & 140 & 155 & 1328 & 137 & 138 & 130 & 635 & 135 & 144 & 161 & 1601 \\
\hline 1 & 177 & 173 & 142 & 227 & 152 & 163 & 183 & 2162 & 187 & 196 & 212 & 2031 \\
\hline 2 & 160 & 172 & 190 & 1624 & 184 & 195 & 210 & 1801 & 162 & 168 & 173 & 710 \\
\hline 3 & 180 & 190 & 205 & 2096 & 160 & 164 & 169 & 453 & 170 & 180 & 197 & 1879 \\
\hline 4 & 171 & 174 & 173 & 261 & 174 & 184 & 199 & 941 & 190 & 188 & 165 & 814 \\
\hline 5 & 198 & 208 & 221 & 2864 & 192 & 190 & 169 & 669 & 206 & 211 & 213 & 5831 \\
\hline 6 & 192 & 191 & 169 & 671 & 205 & 210 & 212 & 5556 & 204 & 204 & 193 & 990 \\
\hline 7 & 206 & 209 & 208 & 2667 & 204 & 204 & 192 & 829 & 215 & 219 & 222 & 11704 \\
\hline 8 & 205 & 205 & 192 & 750 & 214 & 219 & 222 & 12514 & & & & \\
\hline 9 & 215 & 218 & 221 & 13072 & & & & & & & & \\
\hline
\end{tabular}

Table 4. The result of clustering image for round 10, 11 and 12

\begin{tabular}{|c|c|c|c|c|c|c|c|c|c|c|c|c|}
\hline \multirow[t]{2}{*}{$\begin{array}{l}\text { Cluster } \\
\text { Number }\end{array}$} & $\begin{array}{c}\text { Center } \\
\text { Red }\end{array}$ & $\begin{array}{l}\text { Center } \\
\text { Green }\end{array}$ & $\begin{array}{c}\text { Center } \\
\text { Blue }\end{array}$ & \multirow[t]{2}{*}{ Count } & $\begin{array}{c}\text { Center } \\
\text { Red }\end{array}$ & $\begin{array}{l}\text { Center } \\
\text { Green }\end{array}$ & $\begin{array}{c}\text { Center } \\
\text { Blue }\end{array}$ & Count & $\begin{array}{c}\text { Center } \\
\text { Red }\end{array}$ & $\begin{array}{l}\text { Center } \\
\text { Green }\end{array}$ & $\begin{array}{c}\text { Center } \\
\text { Blue }\end{array}$ & \multirow[t]{2}{*}{ Count } \\
\hline & \multicolumn{3}{|c|}{ Number Round $=10$} & & \multicolumn{4}{|c|}{ Number Round=11 } & \multicolumn{3}{|c|}{ Number Round $=12$} & \\
\hline 1 & 142 & 151 & 165 & 2227 & 166 & 177 & 194 & 2312 & & & & \\
\hline 2 & 169 & 179 & 197 & 1776 & 188 & 186 & 163 & 916 & & & & \\
\hline 3 & 189 & 187 & 163 & 831 & 188 & 197 & 211 & 2611 & & & & \\
\hline 5 & 206 & 207 & 200 & 1979 & 213 & 217 & 221 & 15219 & & & & \\
\hline 6 & 213 & 217 & 220 & 15652 & & & & & & & & \\
\hline
\end{tabular}

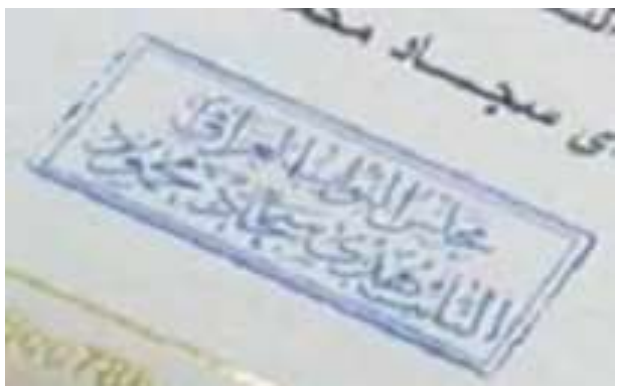

(a) Original Image

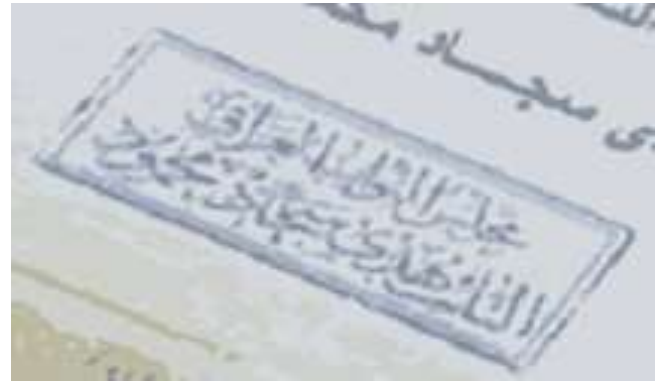

(b) After Clustering

Figure 2. Image clustering using K-mean \& ISODATA 
Table 5. Result of merge clusters

\begin{tabular}{cccccccc}
\hline $\begin{array}{c}\text { Cluster } \\
\text { Merge }\end{array}$ & $\begin{array}{c}\text { Cluster } \\
\text { Merge } \\
\text { with }\end{array}$ & $\begin{array}{c}\text { New } \\
\text { Cluster } \\
\text { Number }\end{array}$ & $\begin{array}{c}\text { Minimum } \\
\text { Distance }\end{array}$ & $\begin{array}{c}\text { Center } \\
\text { Red }\end{array}$ & $\begin{array}{c}\text { Center } \\
\text { Green }\end{array}$ & $\begin{array}{c}\text { Center } \\
\text { Blue }\end{array}$ & Count \\
\hline 4 & 5 & $\mathbf{0}$ & 33 & 71 & 72 & 73 & 54225 \\
2 & 3 & $\mathbf{1}$ & 59 & 63 & 65 & 66 & 10581 \\
0 & 1 & $\mathbf{2}$ & 97 & 51 & 54 & 60 & 11874 \\
\hline
\end{tabular}

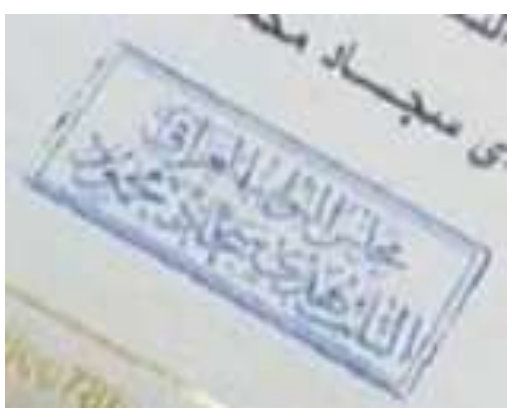

(a) Original image

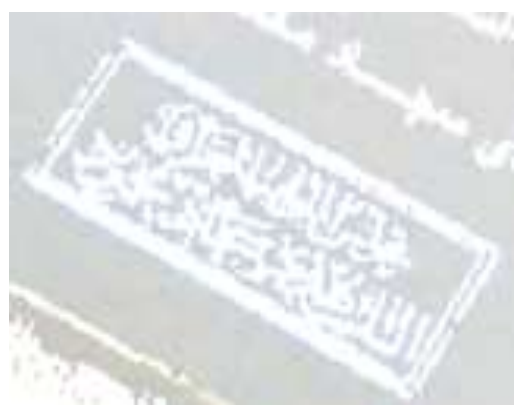

(b) Background

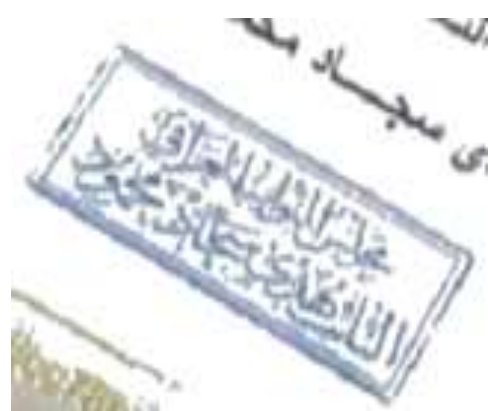

(c) Stamp with noise

Figure 3. Isolated background cluster from stamp cluster

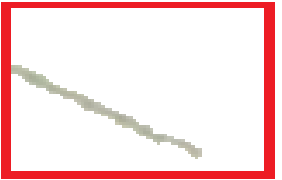

Segment0

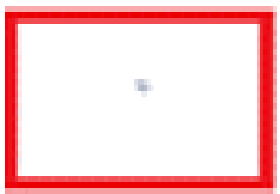

Segment5

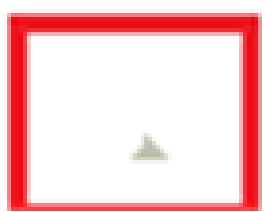

Segment10

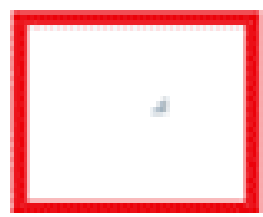

Segment15

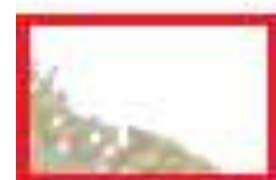

Segment1

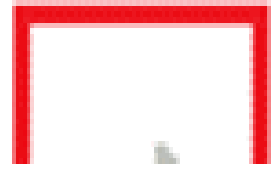

Segment6

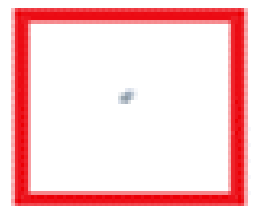

Segment11

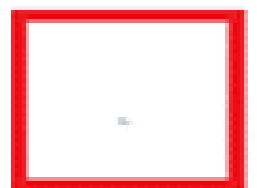

Segment 16

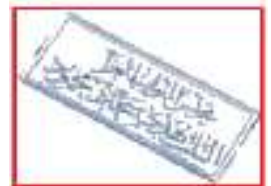

Segment2

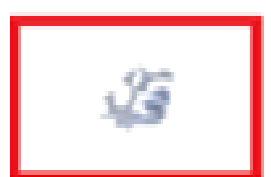

Segment7

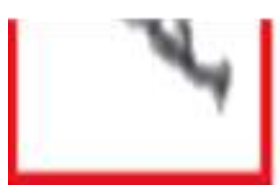

Segment12

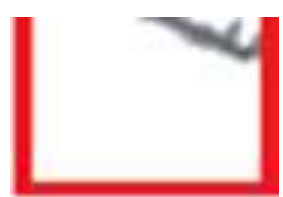

Segment 17

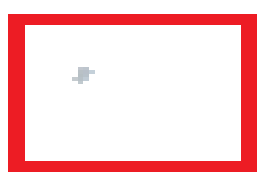

Segment3

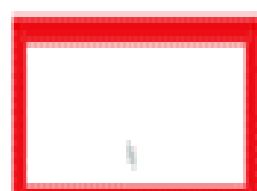

Segment8

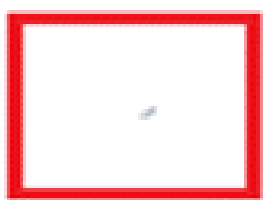

Segment13

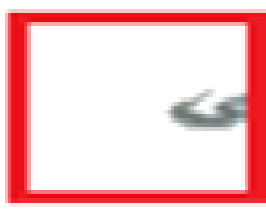

Segment 18

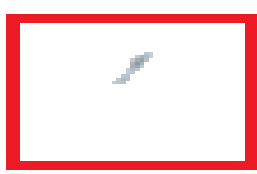

Segment4

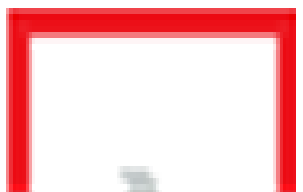

Segment9

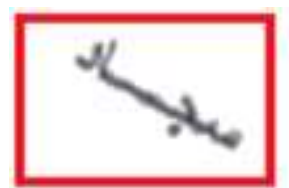

Segment14

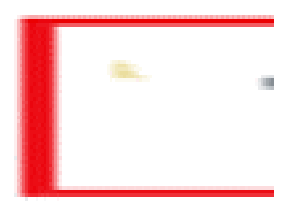

Segment19

Figure 4. The segments of image after applying segmentation

Figure 5 explains the result of extraction stamp image and remove noise for different classes chosen randomly from the dataset. 


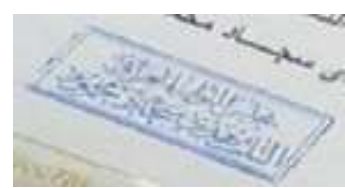

(a): Original Image

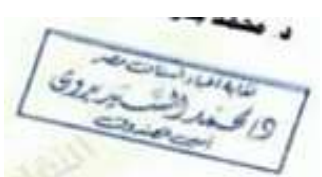

(a): Original Image

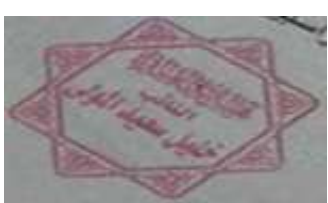

(a): Original Image

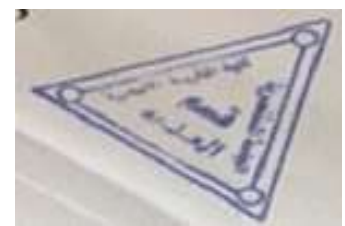

(a): Original Image

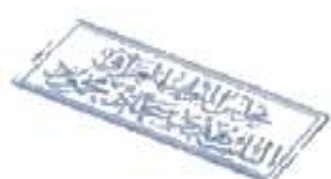

(b): Stamp Image

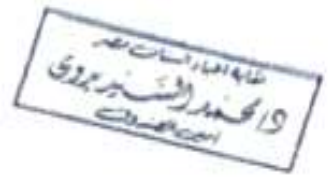

(b): Stamp Image

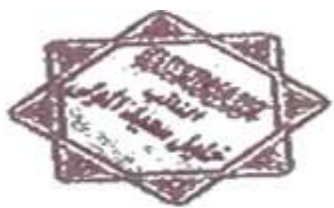

(b): Stamp Image

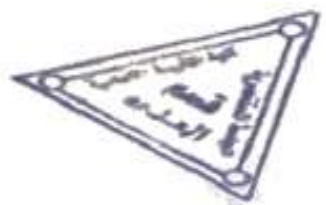

(b): Stamp Image

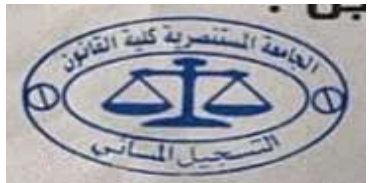

(a): Original Image

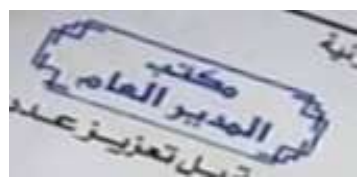

(a): Original Image

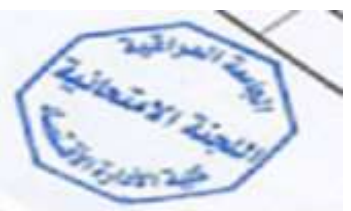

(a): Original Image

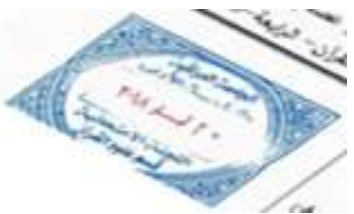

(a): Original Image

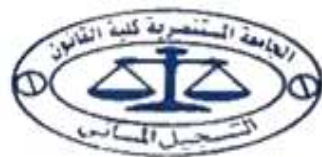

(b): Stamp Image

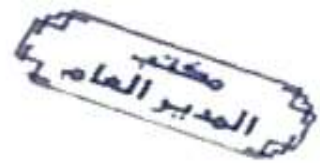

(b): Stamp Image

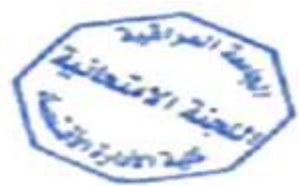

(b): Stamp Image

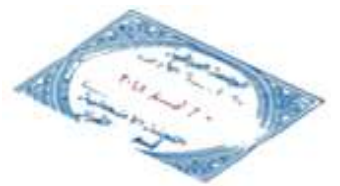

(b): Stamp Image

Figure 5. The results of extraction stamp image for different classes

\section{CONCLUSION}

This paper presents an efficient and robust method for extracting stamp and removing unwanted data. The method includes the use image clustering techniques such as k-mean clustering algorithm and ISODATA clustering algorithm to cluster the data points into a number of clusters and to isolate the background cluster from stamp cluster and compute mean and standard deviation for the resulting clusters. Also, it includes the use of image segmentation techniques such as region growing algorithm based on eight neighbors to segment the image into a number of segments and chosen the connected segment to extract stamp and removing noise. To extraction stamp, a set of points is computed from each segment to produce a binary mask and to multiply it by an original image to produced stamp free from noise. This step is very important to extract all useful feature for stamp recognition system. The results achieved indicated that the proposed method was promising, fast and very appropriate as a preliminary stage in image recognition applications.

\section{REFERENCES}

[1] B. Micenkova and J. Beusekom, "Stamp detection in color document images," International Conference on Document Analysis and Recognition, pp. 1125-1129, 2011.

[2] P. Forczman'ski and D. Frejlichowski," Robust stamps detection and classification by means of general shape analysis," Springer International Conference on Computer Vision and Graphics, pp. 1-8, 2010.

[3] P. Gantuya, et al., " Mongolian traditional stamp recognition using scalable kNN, " International Journal of Advanced Smart Convergence, vol. 4, no. 2, pp. 170-176, 2015.

[4] A. V. Nandedkar, et al., "A spectral filtering based deep learning for detection of logo and stamp, " IEEE Conference: Fifth National Conference on Computer Vision, Pattern Recognition, Image Processing and Graphics (NCVPRIPG), 2015.

[5] P. Forczmański, "Stamp detection in scanned documents," Annales UMCS Informatica Lublin-Polonia Sectio AI, vol. 10, no. 1, pp. 61-68, 2010.

[6] P. Forczmański and D. Frejlichowski, "Classification of elementary stamp shapes by means of reduced point distance histogram representation," Springer-Verlag Berlin Heidelberg, pp. 603-616, 2012. 
[7] Dey, Soumyadeep, Jayanta Mukhopadhyay, and Shamik Sural," Removal of gray rubber stamps," IAPR Workshop on Document Analysis Systems, pp. 210- 214, 2016.

[8] A. V. Nandedkar, et al., "A spectral filtering based deep learning for detection of logo and stamp," IEEE Conference: Fifth National Conference on Computer Vision, Pattern Recognition, Image Processing and Graphics (NCVPRIPG), 2015.

[9] A. V. Nandedkar, et al., "SPODS: A dataset of color-official documents and detection of logo, stamp, and signature," nternational Conference on Computer Vision, Graphics, and Image processing, pp. 219-230, 2017.

[10] A. Farahmand, et al., "Document image noises and removal methods," International MultiConference of Engineers and Computer Scientists (IMECS), vol. 1, 2013.

[11] A. Vijayalakshmi, et al., "Image denoising for different noise models by various filters: A brief survey," International Journal of Emerging Trends \& Technology in Computer Science (IJETTCS), vol. 3, no. 6, pp. 42-45, 2014.

[12] G. K. Seerha and R. Kaur, "Review on recent image segmentation techniques," International Journal on Computer Science and Engineering (IJCSE), vol. 5, no. 02, pp. 109-112, 2013.

[13] R. Kandwal, et al., "Review: Existing image segmentation techniques," International Journal of Advanced Research in Computer Science and Software Engineering, vol.4, no. 4, pp. 153-156, 2014.

[14] K. B. Kim, et al., "Automatic segmentation of wrist bone fracture area by k-means pixel clustering from x-ray image," International Journal of Electrical and Computer Engineering (IJECE), vol. 9, no. 6, pp. 5205-5210, 2019.

[15] S. Panda, "Color image segmentation using k-means clustering and thresholding technique, " Interntional journal of ESC, pp.1132-1136, 2015.

[16] N. M. Mahfuz, et al., "Review of single clustering methods, " IAES International Journal of Artificial Intelligence (IJ-AI), vol. 8, no. 3, pp. 221-227, 2019.

[17] P. Panwar, et al., "Image segmentation using k-means clustering and thresholding, " International Research Journal of Engineering and Technology (IRJET), vol. 03, no. 05, pp. 1787-1793, 2016.

[18] N. Dhanachandra, et al., "Image segmentation using $k$-means clustering algorithm and subtractive clustering algorithm," Elsevier Eleventh International Multi-Conference on Information Processing (IMCIP), vol. 54, pp. 764-771, 2015.

[19] Y. Hamzaoui, et al., "Enhancenig OLSR routing protocol using k-means clustering in MANETs," International Journal of Electrical and Computer Engineering (IJECE), vol. 10, no. 4, pp. 3715-3724, 2020.

[20] S. Singh and P. Singh, "Speaker specific feature based clustering and its applications in language independent forensic speaker recognition, "International Journal of Electrical and Computer Engineering (IJECE), vol. 10, no. 4, pp. 3508-3518, 2020.

[21] W. Liu, et al., "An adaptive clustering algorithm based on the possibility clustering and ISODATA for multispectral image classification," The International Archives of the Photogrammetry, Remote Sensing and Spatial Information Sciences, vol. 37, pp. 565- 568, 2008.

[22] M. Merzougui, et al., "Image segmentation using isodata clustering with parameters estimated by evolutionary approach: application to quality control," International Journal of Computer Applications, vol. 66, no. 19, pp. 25$30,2013$.

[23] A. W. Abbas, et al., "K-Means and ISODATA clustering algorithms for landcover classification using remote sensing," Sindh University Research Journal (Science Series), vol. 48, no. 2, pp. 315-318, 2016.

[24] Q. Lu, et al., "A novel clustering-based feature representation for the classification of hyperspectral imagery," Remote Sensing, vol. 6, no. 6, pp. 5732-5753, 2014.

[25] M. N. Qureshia and M. V. Ahamadb, "An improved method for image segmentation using k-means clustering with neutrosophic logic, " Elsevier. International Conference on Computational Intelligence and Data Science (ICCIDS), vol. 132, pp. 534-540, 2018. 